\title{
Using clinical guidelines to assess the potential value of laboratory medicine in clinical decision-making
}

\author{
Allan J. Hicks ${ }^{1}$, Zoe L. Carwardine ${ }^{2}$, Mike J. Hallworth ${ }^{3}$, Eric S. Kilpatrick*4 \\ ${ }^{1}$ School of Medical Science, Griffith University, Southport, Australia \\ ${ }^{2}$ Clinical Biochemistry, Sidra Medicine, Doha, Qatar \\ ${ }^{3}$ Clinical Biochemistry, Royal Shrewsbury Hospital, Shrewsbury, UK \\ ${ }^{4}$ Department of Clinical Biochemistry, Manchester Royal Infirmary, Manchester University NHS Foundation Trust, Manchester, UK \\ *Corresponding author: Eric.Kilpatrick@mft.nhs.uk
}

\begin{abstract}
Introduction: It is often quoted that $70 \%$ of clinical decisions are based on laboratory results, but the evidence to substantiate this claim is lacking. Since clinical guidelines aim to document best-practice decision making for specific disease conditions, inclusion of any laboratory test means that the best available evidence is recommending clinicians use it. Cardiovascular disease (CVD) is the world's most common cause of mortality, so this study reviewed all CVD guidelines published by five national/international authorities to determine what proportion of them recommended laboratory testing.
\end{abstract}

Materials and methods: Five leading CVD guidelines were examined, namely the European Society of Cardiology (ESC), the UK National Institute for Health and Clinical Excellence (NICE), the American College of Cardiology (ACC), the Australian Heart Foundation (AHF) and the Cardiac Society of Australia and New Zealand (CSANZ).

Results: A total of 101 guidelines were reviewed. Of the 33 individual ESC guidelines relating to CVD, 24/33 made a direct reference to the use of clinical laboratory tests in either diagnosis or follow-up treatment. The same applied to 15/20 of NICE guidelines, 24/32 from the ACC and 15/16 from the AHF/CSANZ. Renal function and blood count testing were the most recommended (39 and 26 times), with lipid, troponin and natriuretic peptide measurement advocated 25,19 and 19 times respectively.

Conclusions: This study has shown that laboratory testing is advocated by between $73 \%$ and $94 \%$ of individual CVD guideline recommendations from five national/international authorities. This provides an index to assess the potential value of laboratory medicine to healthcare.

Keywords: cardiovascular diseases; guideline; clinical laboratory testing

\section{Introduction}

The contribution of laboratory medicine to patient diagnosis, management and follow-up has proven difficult to quantify with systematic evidence of improved patient outcomes scarce (1). The phrase that 'laboratory medicine influences $70 \%$ of clinical decisions', or similar, has been published many times but the evidence to substantiate this claim is lacking. An editorial in the Annals of Clinical Biochemistry stated that the $70 \%$ figure was first published in 1996 and was based on anecdotal evi- dence and unpublished studies (2). The editorial lists various examples of the use of this phrase, albeit with slight modifications, for example; Lord Carter's report on the UK Pathology service, the First Report of the UK House of Commons Select Committee on Health, and in the UK Department of Health report "Modernizing Pathology Services" (2). A related and also oft-quoted statistic is that $70 \%$ of the electronic patient record is composed of laboratory data, but the main limitation of this obser- 
vation is that the presence of laboratory results in a patient record does not necessarily equate to it being used in any clinical decision-making process (3). Over-requesting of testing is a common feature of many healthcare systems and panels of tests may include many analytes which are unrelated to the patient's clinical condition. A 2016 study of laboratory use by oncologists and cardiologists found that $75 \%$ of all their patients underwent laboratory testing, and that this testing led to a substantial clinical decision in $66 \%$ of the patients (4).

Over time the $70 \%$ claim has apparently gained legitimacy simply due to the number of times that it had been repeated. Partly to examine this claim more closely, the International Federation of Clinical Chemistry and Laboratory Medicine (IFCC) established a Task Force on the Impact of Laboratory Medicine on Clinical Management and Outcomes in 2012. Its purpose was twofold: first, to evaluate the available evidence supporting the impact of laboratory medicine in healthcare; and secondly to develop a study design methodology for new retrospective and prospective studies capable of generating evidence to determine the contribution made by laboratory medicine to healthcare.

The IFCC Task Force published a summary of its findings in 2015 which included as one of its suggestions that the contents of authoritative clinical guidelines could provide an objective means of assessing the role of laboratory medicine in the management of specific health conditions (1). Clinical guidelines are documents which aim to guide decisions regarding diagnosis, management and treatment in specific areas of health care. By using the best evidence available the assumption is that each individual guideline recommendation is how clinicians should be making their clinical decisions.

In respect to which health condition should have its guidelines examined, according to the World Health Organization (WHO) cardiovascular disease (CVD) is the world's most prevalent cause of mortality encompassing a large number of diseases including those of coronary heart disease, heart failure, rhythm and valvular abnormalities as well as cerebrovascular diseases (5). The report estimated that 17.9 million people worldwide die from CVDs which equates to $31 \%$ of all deaths and, of these,
$85 \%$ were due to myocardial infarction or stroke (5). The British Heart Foundation report that CVD accounts for almost 170,000 deaths in the UK costing the National Health Service there $£ 6.8$ billion in 2012/2013 (6). In Europe, CVD is responsible for 3.9 million (45\%) of all deaths annually (7). The American Heart Association has reported that coronary heart disease is the leading cause of death among Americans, accounting for 840,678 (30\%) deaths in 2016 (8). According to the Australian Institute of Health and Welfare Alliance, CVD directly contributed to 45,400 deaths in 2015 which accounts for $29 \%$ of all deaths that year in Australia (9).

Accordingly, this study has chosen to examine some national and international cardiovascular clinical guidelines in detail from the countries just mentioned in order to determine what proportion of them recommended laboratory testing.

\section{Materials and methods}

We conducted a review of all the individual cardiovascular guidelines available on the websites of the UK National Institute for Health and Care Excellence (NICE), the European Society of Cardiology (ESC), the American College of Cardiology (ACC), and Cardiac Society of Australia and New Zealand (CSANZ) and Australian Heart Foundation (AHF) in their online versions which were current on the 23rd April 2020 (10-14). All the guidelines from the cardiology societies were examined while the NICE guidelines were limited only to those categorised by them as referring to 'cardiovascular conditions'. The AHF and the CSANZ guidelines were combined because a number of their guidelines were shared and, where they were identical, only one was included in the analysis statistics. These specific national/international guidelines were chosen because of their geographical spread and because they have previously been found to be rigorous with, for example, NICE and ESC guidance having the highest Appraisal of Guidelines for Research and Evaluation (AGREE) II scores for cardiovascular risk assessment in a systematic review (15).

Each individual guideline was read through in its entirety to determine if it was documented that laboratory involvement (defined as a test usually 
associated with one of the Laboratory Medicine disciplines, Histology or Genetics) was required for either an initial diagnosis or the ongoing management of care of the cardiovascular condition referred to in the document. Data was collected separately for tests which were used for initial diagnosis compared to those required for follow-up, and all forms of testing were included, not just analyses traditionally associated with CVD.

The proportion of guidelines containing laboratory medicine recommendations were determined for each national/international authority (AHF and CSANZ regarded as one) and for them combined. These proportions were calculated by simply dividing the number of individual guidelines indicating testing (if used for diagnosis and/or followup this was counted as one) by the total number of guidelines, whether for each national/international authority or for all authorities combined.

For solely the purpose of determining which types of tests were mentioned most frequently by the guidelines, some related tests were categorised together. Thus, renal function related testing included 'renal function tests', 'creatinine' and 'eGFR' in the text; natriuretic peptides included 'natriuretic peptides', 'BNP' and 'NT-proBNP'; 'calcium', if mentioned separately from other electrolytes, was included in an "electrolytes group" and FBC and $C B C$ were regarded as synonyms. Genetic and histology tests were not subcategorised any further.

As this is a review of existing guidelines, no Institutional Review Board approval was required.

\section{Results}

Table 1 shows the 101 guidelines related to CVD available from NICE, ESC, ACC and the AHF/CSANZ and summarises the number and/or percentage indicating pathology testing for either initial diagnosis, follow up pathology testing or a combination of both. Taken together, in vitro diagnostic testing was required for diagnosing $64 \%$ of the conditions alluded to in the cardiovascular guidelines, with a similar proportion (62\%) advocating testing for the continued management of such patients. As tests were sometimes recommended for both the diagnosis and follow-up of same condition, it means some form of testing was, on average, required by $77 \%$ of individual guidelines.

Tables 2-5 respectively show details of the individual NICE, ESC, ACC and the AHF/CSANZ guidelines including the year published and the tests mentioned within the body of the document. The test names used in these tables are as described in the respective guidelines.

Twenty seven different test categories were explicitly mentioned 276 times within the 78 guidelines requiring testing (Tables $2-5$ ). The commonest were renal function related biochemistry (mentioned 39 times), complete (or 'full') blood counts (26 times), lipids (25 times) and blood/plasma glucose (20 times). More specific to cardiovascular disease were the troponins (19 times) and natriuretic peptides (19 times).

TABLE 1. Summary of requirement for in vitro diagnostic testing as part of individual cardiovascular guideline recommendations from 5 sources

\begin{tabular}{lcccc}
\hline $\begin{array}{l}\text { Organisation } \\
\text { (Country/Region) }\end{array}$ & $\begin{array}{c}\text { Testing required for } \\
\text { initial diagnosis* }\end{array}$ & $\begin{array}{c}\text { Testing required } \\
\text { for further } \\
\text { management }\end{array}$ & $\begin{array}{c}\text { Guidelines requiring } \\
\text { any testing }\end{array}$ & $\begin{array}{c}\text { Total number of CVD } \\
\text { guidelines }\end{array}$ \\
\hline NICE (UK) & 10 & 13 & 15 & 20 \\
\hline ESC (Europe) & 20 & 17 & 24 & 33 \\
\hline ACC (USA) & 20 & 24 & 24 & 32 \\
\hline AHF/CSANZ (Australasia) & 15 & 9 & 15 & 16 \\
\hline Total, N (\%) & $65(64)$ & $63(62)$ & $78(77)$ & $101(100)$ \\
\hline
\end{tabular}

*All refer to the number of guidelines requiring testing. NICE - UK National Institute for Health and Clinical Excellence. ESC European Society of Cardiology. ACC - American College of Cardiology. AHF - Australian Heart Foundation. CSANZ - Cardiac Society of Australia and New Zealand. CVD - cardiovascular disease. 
TABLE 2. National Institute for Health and Clinical Excellence (NICE) Guidelines

\begin{tabular}{|c|c|c|c|c|}
\hline Guideline & $\begin{array}{l}\text { Year } \\
\text { published }\end{array}$ & $\begin{array}{l}\text { Testing required } \\
\text { for initial } \\
\text { diagnosis }\end{array}$ & $\begin{array}{l}\text { Testing required } \\
\text { for further } \\
\text { management }\end{array}$ & Indicated test(s) \\
\hline $\begin{array}{l}\text { Hypertension in pregnancy: diagnosis and } \\
\text { management }\end{array}$ & 2019 & Yes & Yes & RFT, LFT, FBC, urinalysis \\
\hline $\begin{array}{l}\text { Chronic heart failure in adults: } \\
\text { management }\end{array}$ & 2018 & Yes & Yes & $\begin{array}{l}\text { NT-proBNP, RFT, LFT, } \\
\text { FBC, lipids, HbA1c, TFT, } \\
\text { urinalysis }\end{array}$ \\
\hline Stable angina: management & 2016 & Yes & Yes & cTn, FBC \\
\hline $\begin{array}{l}\text { Hypertension in adults: diagnosis and } \\
\text { management }\end{array}$ & 2019 & No & Yes & $\begin{array}{l}\text { RFT, eGFR, Lipids, HbA1c, } \\
\text { creatinine, urinalysis }\end{array}$ \\
\hline $\begin{array}{l}\text { Hyperglycaemia in acute coronary } \\
\text { syndromes: management }\end{array}$ & 2016 & No & Yes & Glucose, $\mathrm{HbA1c}$ \\
\hline $\begin{array}{l}\text { Venous thromboembolic diseases: } \\
\text { diagnosis, management and thrombophilia } \\
\text { testing }\end{array}$ & 2020 & Yes & Yes & $\begin{array}{l}\text { D-dimer, PT, APTT, FBC, } \\
\text { RFT, LFT }\end{array}$ \\
\hline $\begin{array}{l}\text { Peripheral arterial disease: diagnosis and } \\
\text { management }\end{array}$ & 2018 & No & No & / \\
\hline Stroke rehabilitation in adults & 2013 & No & No & / \\
\hline $\begin{array}{l}\text { Myocardial infarction with ST-segment } \\
\text { elevation: acute management }\end{array}$ & 2013 & No & No & / \\
\hline Varicose veins: diagnosis and management & 2013 & No & No & / \\
\hline Atrial fibrillation & 2014 & No & Yes & $\begin{array}{l}\text { LFT, INR, digoxin } \\
\text { concentrations }\end{array}$ \\
\hline $\begin{array}{l}\text { Cardiovascular Disease: risk assessment and } \\
\text { reduction, including lipid modification }\end{array}$ & 2016 & Yes & Yes & $\begin{array}{l}\text { eGFR, lipids, albumin, } \\
\text { HbA1c, LFT, RFT, CK }\end{array}$ \\
\hline $\begin{array}{l}\text { Acute Heart Failure: diagnosis and } \\
\text { management }\end{array}$ & 2014 & Yes & Yes & BNP, RFT, NT-proBNP \\
\hline $\begin{array}{l}\text { Prophylaxis against infective endocarditis: } \\
\text { antimicrobial prophylaxis against infective } \\
\text { endocarditis in adults and children } \\
\text { undergoing interventional procedures }\end{array}$ & 2016 & No & No & / \\
\hline $\begin{array}{l}\text { Stroke and transient ischaemic attack in } \\
\text { over 16s: diagnosis and initial management }\end{array}$ & 2019 & No & Yes & INR, glucose, HbA1c, \\
\hline $\begin{array}{l}\text { Familial hypercholesterolemia: } \\
\text { identification and management }\end{array}$ & 2019 & Yes & Yes & Lipids \\
\hline $\begin{array}{l}\text { Venous thromboembolism in over 16s: } \\
\text { reducing the risk of hospital-acquired deep } \\
\text { vein thrombosis or pulmonary embolism }\end{array}$ & 2019 & No & No & eGFR \\
\hline $\begin{array}{l}\text { Unstable angina and Non-ST-elevation } \\
\text { myocardial infarction: early management }\end{array}$ & 2013 & No & Yes & $\begin{array}{l}\text { cTn, creatinine, glucose, } \\
\text { FBC }\end{array}$ \\
\hline $\begin{array}{l}\text { Recent-onset chest pain of suspected } \\
\text { cardiac origin: assessment and diagnosis }\end{array}$ & 2016 & Yes & No & cTn \\
\hline $\begin{array}{l}\text { Myocardial Infarction: cardiac rehabilitation } \\
\text { and prevention of further } \mathrm{MI}\end{array}$ & 2013 & No & Yes & RFT \\
\hline
\end{tabular}

RFT - renal function tests. LFT - liver function tests. FBC - full blood count. NT-proBNP - N-terminal pro-B-type natriuretic peptide. HbA1c - haemoglobin A1c. TFT - thyroid function tests. cTn - cardiac troponins. eGFR - estimated glomerular filtration rate. PT prothrombin time. APTT - activated partial thromboplastin time. INR - international normalized ratio. CK - creatine kinase. BNP B-type natriuretic peptide. 
TABLE 3. European Society of Cardiology (ESC) guidelines

\begin{tabular}{|c|c|c|c|c|}
\hline Guidelines & $\begin{array}{l}\text { Year } \\
\text { published }\end{array}$ & $\begin{array}{l}\text { Testing required } \\
\text { for initial } \\
\text { diagnosis }\end{array}$ & $\begin{array}{l}\text { Testing required } \\
\text { for further } \\
\text { management }\end{array}$ & Indicated test(s) \\
\hline $\begin{array}{l}\text { Infective Endocarditis (Guidelines on } \\
\text { Prevention, Diagnosis and Treatment of) }\end{array}$ & 2015 & Yes & Yes & $\begin{array}{c}\text { CRP, ESR, IS, BC, creatinine, } \\
\text { bilirubin, } C B C\end{array}$ \\
\hline $\begin{array}{l}\text { Ventricular Arrhythmias and the Prevention } \\
\text { of Sudden Cardiac Death }\end{array}$ & 2015 & No & Yes & Electrolytes, \\
\hline $\begin{array}{l}\text { Pericardial Diseases (Guidelines on the } \\
\text { Diagnosis and Management of) }\end{array}$ & 2015 & Yes & Yes & $\begin{array}{c}\text { CRP, CBC, ESR, CK, cTn, RFT, } \\
\text { LFT }\end{array}$ \\
\hline $\begin{array}{l}\text { Acute Coronary Syndromes in patients } \\
\text { presenting without persistent ST-segment } \\
\text { elevation }\end{array}$ & 2015 & Yes & Yes & cTn, lipids \\
\hline $\begin{array}{l}\text { Pulmonary Hypertension (Guidelines on } \\
\text { Diagnosis and Treatment of) }\end{array}$ & 2015 & Yes & Yes & $\mathrm{RFT}, \mathrm{CBC}$, iron studies, LFT \\
\hline Hypertrophic Cardiomyopathy & 2014 & Yes & No & BNP, cTn, CK, TFT, RFT, CBC, \\
\hline Aortic Diseases & 2014 & Yes & No & BNP, cTn \\
\hline $\begin{array}{l}\text { ESC/EACTS Guidelines in Myocardial } \\
\text { Revascularisation (Guidelines for) }\end{array}$ & 2018 & No & No & / \\
\hline $\begin{array}{l}\text { Acute Pulmonary Embolism (Diagnosis and } \\
\text { Management of) }\end{array}$ & 2019 & Yes & No & D-dimer \\
\hline $\begin{array}{l}\text { ESC/ESA Guidelines on non-cardiac } \\
\text { surgery: cardiovascular assessment and } \\
\text { management }\end{array}$ & 2014 & Yes & No & cTn \\
\hline $\begin{array}{l}\text { Diabetes, Pre-Diabetes and Cardiovascular } \\
\text { Diseases developed with the EASD }\end{array}$ & 2019 & Yes & Yes & Glucose, lipids, HbA1c \\
\hline $\begin{array}{l}\text { Stable Coronary Artery Disease } \\
\text { (Management of) }\end{array}$ & 2019 & Yes & Yes & $\begin{array}{c}\text { Glucose, LFT, TFT, CBC, lipids, } \\
\text { HbA1c, CK, creatinine }\end{array}$ \\
\hline $\begin{array}{l}\text { Cardiac Pacing and Cardiac } \\
\text { Resynchronization Therapy }\end{array}$ & 2013 & No & No & \\
\hline Arterial Hypertension (Management of) & 2018 & Yes & Yes & $\begin{array}{l}\text { Glucose, LFT, RFT, eGFR, } \\
\text { lipids, creatinine }\end{array}$ \\
\hline Valvular Heart Disease (Management of) & 2017 & Yes & Yes & BNP \\
\hline $\begin{array}{l}\text { Atrial Fibrillation (Management of) } 2010 \\
\text { and Focused Update (2012) }\end{array}$ & 2016 & No & No & / \\
\hline $\begin{array}{l}\text { Acute Myocardial Infarction in patients } \\
\text { presenting with ST-segment elevation } \\
\text { (Management of) }\end{array}$ & 2017 & Yes & No & cTn \\
\hline Acute and Chronic Heart Failure & 2016 & Yes & Yes & BNP \\
\hline $\begin{array}{l}\text { CVD Prevention in clinical practice } \\
\text { (European Guidelines on) }\end{array}$ & 2016 & No & No & / \\
\hline Dyslipidaemias (Management of) & 2019 & Yes & Yes & Lipids, CRP \\
\hline $\begin{array}{l}\text { Cardiovascular Diseases during Pregnancy } \\
\text { (Management of) }\end{array}$ & 2018 & Yes & Yes & $\begin{array}{l}\text { CBC, RFT, LFT, BNP, cTn, } \\
\text { D-dimer, urine protein }\end{array}$ \\
\hline $\begin{array}{l}\text { Peripheral Artery Diseases (Diagnosis and } \\
\text { Treatment of) }\end{array}$ & 2017 & Yes & Yes & $\begin{array}{c}\text { Glucose, lipids, creatinine, } \\
\text { urine protein, CBC, RFT, } \\
\text { HbA1c, }\end{array}$ \\
\hline $\begin{array}{l}\text { Grown-Up Congenital Heart Disease } \\
\text { (Management of) }\end{array}$ & 2010 & No & Yes & $\begin{array}{l}\text { CBC, ferritin, creatinine, uric } \\
\text { acid, BNP, folic acid, vitamin } \\
\text { B12 }\end{array}$ \\
\hline
\end{tabular}


TABle 3. Continued

\begin{tabular}{|c|c|c|c|c|}
\hline Guidelines & $\begin{array}{c}\text { Year } \\
\text { published }\end{array}$ & $\begin{array}{l}\text { Testing required } \\
\text { for initial } \\
\text { diagnosis }\end{array}$ & $\begin{array}{l}\text { Testing required } \\
\text { for further } \\
\text { management }\end{array}$ & Indicated test(s) \\
\hline $\begin{array}{l}\text { Device Therapy in Heart Failure (Focused } \\
\text { Update) }\end{array}$ & 2010 & No & No & / \\
\hline $\begin{array}{l}\text { Syncope (Guidelines on Diagnosis and } \\
\text { Management of) }\end{array}$ & 2018 & No & No & / \\
\hline $\begin{array}{l}\text { The Role of Endomyocardial Biopsy in the } \\
\text { Management of Cardiovascular Disease }\end{array}$ & 2007 & Yes & No & Histological examination \\
\hline $\begin{array}{l}\text { B-Adrenergic Receptor Blockers (Expert } \\
\text { Consensus Document on) }\end{array}$ & 2004 & No & No & / \\
\hline $\begin{array}{l}\text { Angiotensin Converting Enzyme Inhibitors } \\
\text { in Cardiovascular Disease (Expert } \\
\text { Consensus Document on) }\end{array}$ & 2004 & No & Yes & RFT, creatinine \\
\hline $\begin{array}{l}\text { Antiplatelet Agents (Expert Consensus } \\
\text { Document on the Use of) }\end{array}$ & 2004 & No & No & / \\
\hline $\begin{array}{l}\text { Supraventricular Arrhythmias (ACC/AHA/ } \\
\text { ESC Guidelines for the Management of } \\
\text { Patients with) }\end{array}$ & 2003 & No & Yes & TFT \\
\hline $\begin{array}{l}\text { Estimation of ten-year risk of fatal } \\
\text { cardiovascular disease in Europe: the } \\
\text { SCORE project }\end{array}$ & 2003 & Yes & No & Lipids \\
\hline $\begin{array}{l}\text { Neonatal Electrocardiogram (Guidelines for } \\
\text { the interpretation of the) }\end{array}$ & 2002 & No & No & / \\
\hline Chest Pain (Management of) & 2002 & Yes & Yes & cTn, CK \\
\hline
\end{tabular}

CRP - C-reactive protein. ESR - erythrocyte sedimentation rate. IS - infectious serology. BC - blood culture. CBC - complete blood count. CK - creatine kinase. CTn - cardiac troponins. RFT - renal function tests. LFT - liver function tests. BNP - B-type natriuretic peptide. TFT - thyroid function tests. HbA1c - haemoglobin A1c. eGFR - estimated glomerular filtration rate.

TABLE 4. American College of Cardiology (ACC) Guidelines

\begin{tabular}{|c|c|c|c|c|}
\hline Guideline & $\begin{array}{c}\text { Year } \\
\text { published }\end{array}$ & $\begin{array}{l}\text { Testing required } \\
\text { for initial } \\
\text { diagnosis }\end{array}$ & $\begin{array}{l}\text { Testing required } \\
\text { for further } \\
\text { management }\end{array}$ & Indicated test(s) \\
\hline $\begin{array}{l}\text { Focused Update of the } 2014 \text { AHA/ACC/HRS } \\
\text { Guideline for the Management of Patients } \\
\text { With Atrial Fibrillation }\end{array}$ & 2019 & No & Yes & $\begin{array}{c}\text { CRP, CBC, TFT, RFT, } \\
\text { LFT, electrolytes, INR, } \\
\text { coagulation monitoring }\end{array}$ \\
\hline $\begin{array}{l}\text { Guideline on the Management of Blood } \\
\text { Cholesterol }\end{array}$ & 2018 & Yes & Yes & Lipids \\
\hline $\begin{array}{l}\text { Guideline on the Evaluation and } \\
\text { Management of Patients With Bradycardia } \\
\text { and Cardiac Conduction Delay }\end{array}$ & 2018 & No & No & / \\
\hline $\begin{array}{l}\text { Guideline for the Management of Adults } \\
\text { With Congenital Heart Disease }\end{array}$ & 2018 & No & Yes & APTT, INR, BNP \\
\hline $\begin{array}{l}\text { Guideline for Management of Patients With } \\
\text { Ventricular Arrhythmias and the Prevention } \\
\text { of Sudden Cardiac Death }\end{array}$ & 2017 & Yes & Yes & $\begin{array}{l}\text { BNP, cTn, electrolytes, lipids, } \\
\text { calcium }\end{array}$ \\
\hline
\end{tabular}


TABLE 4. Continued

\begin{tabular}{|c|c|c|c|c|}
\hline Guideline & $\begin{array}{c}\text { Year } \\
\text { published }\end{array}$ & $\begin{array}{l}\text { Testing required } \\
\text { for initial } \\
\text { diagnosis }\end{array}$ & $\begin{array}{l}\text { Testing required } \\
\text { for further } \\
\text { management }\end{array}$ & Indicated test(s) \\
\hline $\begin{array}{l}\text { Prevention, Detection, Evaluation, and } \\
\text { Management of High Blood Pressure in } \\
\text { Adults }\end{array}$ & 2017 & Yes & Yes & $\begin{array}{c}\text { Fasting glucose, CBC, lipids, } \\
\text { creatinine, eGFR, Ca2+, } \\
\text { electrolytes, TFT, urinalysis }\end{array}$ \\
\hline $\begin{array}{l}\text { Focused Update of the } 2013 \text { ACCF/AHA } \\
\text { Guideline for the Management of Heart } \\
\text { Failure }\end{array}$ & 2017 & Yes & Yes & BNP, cTn, eGFR, iron \\
\hline $\begin{array}{l}\text { Focused Update of the } 2014 \text { AHA/ACC } \\
\text { Guideline for the Management of Patients } \\
\text { With Valvular Heart Disease }\end{array}$ & 2017 & Yes & Yes & $\begin{array}{l}\text { BNP, cTn, electrolytes, RFT, } \\
\text { iron }\end{array}$ \\
\hline $\begin{array}{l}\text { Guideline for the Evaluation and } \\
\text { Management of Patients With Syncope }\end{array}$ & 2017 & Yes & Yes & $\begin{array}{l}\text { CBC, electrolytes, BNP, cTn, } \\
\text { glucose }\end{array}$ \\
\hline $\begin{array}{l}\text { Guideline on the Management of Patients } \\
\text { With Lower Extremity Peripheral Artery } \\
\text { Disease }\end{array}$ & 2016 & No & Yes & Fasting glucose, RFT, $\mathrm{HbA} 1 \mathrm{c}$ \\
\hline $\begin{array}{l}\text { Guideline Focused Update on Duration of } \\
\text { Dual Antiplatelet Therapy in Patients With } \\
\text { Coronary Artery Disease }\end{array}$ & 2016 & No & No & / \\
\hline $\begin{array}{l}\text { Focused Update on Primary Percutaneous } \\
\text { Coronary Intervention for Patients With } \\
\text { ST-Elevation Myocardial Infarction }\end{array}$ & 2015 & No & No & / \\
\hline $\begin{array}{l}\text { Surgery for Aortic Dilatation in Patients } \\
\text { With Bicuspid Aortic Valves }\end{array}$ & 2015 & No & No & / \\
\hline $\begin{array}{l}\text { Guideline for the Management of Adult } \\
\text { Patients With Supraventricular Tachycardia }\end{array}$ & 2015 & No & No & / \\
\hline $\begin{array}{l}\text { Guideline for the Management of Patients } \\
\text { With Non-ST-Elevation Acute Coronary } \\
\text { Syndromes }\end{array}$ & 2014 & Yes & Yes & $\begin{array}{c}\text { cTn, BNP, lipids, RFT fasting } \\
\text { glucose, HbA1c, creatinine, } \\
\text { eGFR, }\end{array}$ \\
\hline $\begin{array}{l}\text { Strategies to Enhance Application of } \\
\text { Clinical Practice Guidelines in Patients With } \\
\text { Cardiovascular Disease and Comorbid } \\
\text { Conditions }\end{array}$ & 2014 & No & No & / \\
\hline $\begin{array}{l}\text { Guideline on Perioperative Cardiovascular } \\
\text { Evaluation and Management of Patients } \\
\text { Undergoing Noncardiac Surgery }\end{array}$ & 2014 & No & Yes & Natriuretic peptides \\
\hline $\begin{array}{l}\text { Guideline for the Diagnosis and } \\
\text { Management of Patients With Stable } \\
\text { Ischemic Heart Disease }\end{array}$ & 2012 & Yes & Yes & Lipids, HbA1c, creatinine \\
\hline $\begin{array}{l}\text { Guideline for the Management of Patients } \\
\text { with Atrial Fibrillation }\end{array}$ & 2014 & Yes & Yes & $\begin{array}{l}\text { CRP, CBC, TFT, RFT, } \\
\text { LFT, electrolytes, INR, } \\
\text { coagulation monitoring }\end{array}$ \\
\hline $\begin{array}{l}\text { Guideline for the Management of Patients } \\
\text { with Valvular Heart Disease }\end{array}$ & 2014 & No & Yes & $\begin{array}{l}\text { eGFR, BNP, INR, LFT, BC, IS, } \\
\text { histology, RF }\end{array}$ \\
\hline $\begin{array}{l}\text { Peripheral Arterial Disease (Lower } \\
\text { Extremity, Renal, Mesenteric, and } \\
\text { Abdominal Aortic) }\end{array}$ & 2013 & No & Yes & Fasting glucose, RFT, $\mathrm{HbA} 1 \mathrm{c}$ \\
\hline $\begin{array}{l}\text { Guideline on the Assessment of } \\
\text { Cardiovascular Risk }\end{array}$ & 2013 & Yes & Yes & CRP, creatinine, eGFR \\
\hline
\end{tabular}


TABLE 4. Continued

\begin{tabular}{|c|c|c|c|c|}
\hline Guideline & $\begin{array}{c}\text { Year } \\
\text { published }\end{array}$ & $\begin{array}{l}\text { Testing required } \\
\text { for initial } \\
\text { diagnosis }\end{array}$ & $\begin{array}{l}\text { Testing required } \\
\text { for further } \\
\text { management }\end{array}$ & Indicated test(s) \\
\hline $\begin{array}{l}\text { Guideline for the Management of Heart } \\
\text { Failure }\end{array}$ & 2013 & Yes & Yes & $\begin{array}{l}\text { Fasting glucose, CBC, lipids, } \\
\text { creatinine, eGFR, Ca2+, } \\
\text { electrolytes, TFT, urinalysis }\end{array}$ \\
\hline ST-Elevation Myocardial Infarction & 2012 & Yes & Yes & $\begin{array}{l}\text { Lipids, glucose, HbA1c, cTn, } \\
\text { RFT, coagulation monitoring }\end{array}$ \\
\hline $\begin{array}{l}\text { Guideline for the Diagnosis and Treatment } \\
\text { of Hypertrophic Cardiomyopathy }\end{array}$ & 2011 & Yes & Yes & cTn, genetic testing \\
\hline $\begin{array}{l}\text { Guideline for Coronary Artery Bypass Graft } \\
\text { Surgery }\end{array}$ & 2011 & No & No & / \\
\hline $\begin{array}{l}\text { Guideline for Percutaneous Coronary } \\
\text { Intervention }\end{array}$ & 2011 & Yes & Yes & $\begin{array}{l}\text { Glucose, HbA1c, RFT, cTn, } \\
\text { BNP, iron, lipids }\end{array}$ \\
\hline $\begin{array}{l}\text { Secondary Prevention and Risk Reduction } \\
\text { Therapy for Patients With Coronary and } \\
\text { Other Atherosclerotic Vascular Disease }\end{array}$ & 2011 & Yes & Yes & lipids, RFT, electrolytes, \\
\hline $\begin{array}{l}\text { Cardiovascular Disease Prevention in } \\
\text { Women }\end{array}$ & 2011 & Yes & Yes & $\begin{array}{l}\text { Lipids, glucose, HbA1c, } \\
\text { hormone concentrations }\end{array}$ \\
\hline $\begin{array}{l}\text { Extracranial Carotid and Vertebral Artery } \\
\text { Disease }\end{array}$ & 2011 & Yes & Yes & $\begin{array}{c}\text { CBC, Ca2+, lipids, glucose, } \\
\text { HbA1c }\end{array}$ \\
\hline Thoracic Aortic Disease & 2010 & Yes & Yes & $\begin{array}{c}\text { Genetic testing, IS, BC, } \\
\text { D-dimer, CRP, CBC, } \\
\text { coagulation monitoring, } \\
\text { blood type and screen, } \\
\text { urinalysis }\end{array}$ \\
\hline $\begin{array}{l}\text { Device-Based Therapy of Cardiac Rhythm } \\
\text { Abnormalities }\end{array}$ & 2008 & No & No & / \\
\hline
\end{tabular}

CRP - C-reactive protein. CBC - complete blood count. TFT - thyroid function tests. RFT - renal function tests. LFT - liver function tests. INR - international normalized ratio. APTT - activated partial thromboplastin time. BNP - B-type natriuretic peptide. cTn cardiac troponins. eGFR - estimated glomerular filtration rate. Ca2+ - ionized calcium. HbA1c - haemoglobin A1c. BC - blood culture. IS - infectious serology. RF - rheumatoid factor. IS - infectious serology.

TABLE 5. Australian Heart Foundation (AHF) and the Cardiac Society of Australia and New Zealand (CSANZ) Guidelines

\begin{tabular}{|c|c|c|c|c|c|}
\hline Guideline & Source & $\begin{array}{c}\text { Year } \\
\text { Published }\end{array}$ & $\begin{array}{l}\text { Testing } \\
\text { required for } \\
\text { initial diagnosis }\end{array}$ & $\begin{array}{l}\text { Testing required } \\
\text { for further } \\
\text { management }\end{array}$ & Indicated test(s) \\
\hline $\begin{array}{l}\text { Australian Clinical Guidelines for the } \\
\text { Diagnosis and Management of Atrial } \\
\text { Fibrillation }\end{array}$ & AHF/CSANZ & 2018 & Yes & Yes & $\begin{array}{l}\text { CBC, RFT, TFT, HbA1c, } \\
\text { INR, electrolytes, } \\
\text { lipids }\end{array}$ \\
\hline $\begin{array}{l}\text { Guidelines for the Prevention, Detection, } \\
\text { and Management of Heart Failure in } \\
\text { Australia }\end{array}$ & AHF/CSANZ & 2018 & Yes & Yes & $\begin{array}{l}\text { BNP, genetic testing, } \\
\text { RFT, creatinine, } \\
\text { glucose, CBC }\end{array}$ \\
\hline Coronary Artery Calcium Scoring & CSANZ & 2017 & Yes & No & $\begin{array}{l}\text { eGFR, glucose, } \\
\text { HbA1c, lipids, }\end{array}$ \\
\hline $\begin{array}{l}\text { Clinical Guideline for the diagnosis and } \\
\text { management of hypertension in adults }\end{array}$ & AHF & 2016 & Yes & Yes & $\begin{array}{c}\text { Urinalysis, RFT, } \\
\text { glucose, eGFR, } \\
\text { creatinine, lipids, CBC }\end{array}$ \\
\hline
\end{tabular}


TABLE 5. Continued

\begin{tabular}{|c|c|c|c|c|c|}
\hline Guideline & Source & $\begin{array}{c}\text { Year } \\
\text { Published }\end{array}$ & $\begin{array}{l}\text { Testing } \\
\text { required for } \\
\text { initial diagnosis }\end{array}$ & $\begin{array}{l}\text { Testing required } \\
\text { for further } \\
\text { management }\end{array}$ & Indicated test(s) \\
\hline $\begin{array}{l}\text { Guidelines for the Diagnosis and } \\
\text { Management of Catecholaminergic } \\
\text { Polymorphic Ventricular Tachycardia } \\
\text { (CPVT) }\end{array}$ & CSANZ & 2016 & No & Yes & Genetic testing \\
\hline $\begin{array}{l}\text { Clinical guidelines for the management } \\
\text { of Acute coronary syndrome }\end{array}$ & AHF/CSANZ & 2016 & Yes & Yes & $\begin{array}{l}\text { cTn, lipids, glucose, } \\
\text { HbA1c, eGFR, APTT, } \\
\text { CBC }\end{array}$ \\
\hline $\begin{array}{l}\text { Diagnosis and Management of Familial } \\
\text { Dilated Cardiomyopathy }\end{array}$ & CSANZ & 2016 & Yes & No & CK, genetic testing \\
\hline $\begin{array}{l}\text { Diagnosis and Management of Familial } \\
\text { Hypercholesterolaemia }\end{array}$ & CSANZ & 2016 & Yes & Yes & $\begin{array}{l}\text { Lipids, genetic } \\
\text { testing }\end{array}$ \\
\hline $\begin{array}{l}\text { Update on the Diagnosis and } \\
\text { Management of Familial Long QT } \\
\text { Syndrome }\end{array}$ & CSANZ & 2016 & Yes & No & Genetic testing \\
\hline $\begin{array}{l}\text { Diagnosis and Management of } \\
\text { Hypertrophic Cardiomyopathy }\end{array}$ & CSANZ & 2016 & Yes & No & $\begin{array}{c}\text { Histological } \\
\text { examination, genetic } \\
\text { testing }\end{array}$ \\
\hline $\begin{array}{l}\text { Update on the diagnosis and } \\
\text { management of inherited aortopathies, } \\
\text { including Marfan syndrome }\end{array}$ & CSANZ & 2016 & Yes & No & genetic testing \\
\hline $\begin{array}{l}\text { The routine cardiac assessment of } \\
\text { newborns with Down } \\
\text { syndrome }\end{array}$ & CSANZ & 2016 & No & No & / \\
\hline $\begin{array}{l}\text { Position Statement on the Diagnosis and } \\
\text { Management of Brugada Syndrome }\end{array}$ & CSANZ & 2015 & Yes & No & Genetic testing \\
\hline $\begin{array}{l}\text { Guidelines for the management of } \\
\text { Absolute cardiovascular disease risk }\end{array}$ & AHF/CSANZ & 2012 & Yes & Yes & $\begin{array}{l}\text { Urinalysis, RFT, } \\
\text { glucose, eGFR, } \\
\text { creatinine, lipids, CBC }\end{array}$ \\
\hline $\begin{array}{l}\text { Guideline for prevention, diagnosis and } \\
\text { management of acute rheumatic fever } \\
\text { and rheumatic heart disease }\end{array}$ & AHF/CSANZ & 2012 & Yes & Yes & CBC, CRP, BC, IS, ESR \\
\hline $\begin{array}{l}\text { Guidelines for the diagnosis and } \\
\text { management of Arrhythmogenic Right } \\
\text { Ventricular Cardiomyopathy }\end{array}$ & CSANZ & 2019 & Yes & No & Genetic testing \\
\hline
\end{tabular}

CBC - complete blood count. RFT - renal function tests. TFT - thyroid function tests. HbA1c - haemoglobin A1c. INR - international normalized ratio. BNP - B-type natriuretic peptide. eGFR - estimated glomerular filtration rate. cTn - cardiac troponins. APTT activated partial thromboplastin time. CK - creatine kinase. CRP - C-reactive protein. BC - blood culture. IS - infectious serology. ESR - erythrocyte sedimentation rate.

\section{Discussion}

Guidelines are designed to inform optimum decision-making by clinicians, and therefore provide a measure of the value of laboratory medicine. This study has shown that between $73 \%$ and $94 \%$ of individual UK (NICE), European (ESC), United States
(ACC) and Australasian (AHF/CSANZ) guidelines related to CVD state that laboratory testing of some sort is recommended. The topics of these individual guidelines tend to be related to specific clinical conditions, so it means that, taken together, $77 \%$ 
of clinical recommendation pathways involving each of these cardiovascular diseases require laboratory assistance at some point in their diagnosis or management.

There are both limitations and strengths to using guidelines as a measure of laboratory medicine's contribution to healthcare. One potential limitation is that clinical guidelines are not necessarily followed by all physicians or other healthcare staff. A clinician's own pathway for patients may therefore involve more or less testing than is being recommended. However, the main advantage of our method is that at least the guidelines define an evidence-based recommendation for best practice in each specific clinical scenario. The guidelines also tend to be specific in the test or tests that are recommended, which is advantageous in two ways. First, it helps ensure that the contribution of laboratory medicine to healthcare is not exaggerated by over-requesting - as could be the case if health records were solely being examined - and, secondly, that a clinical decision relevant to the health condition is intended to be taken on the basis of the result.

It should be noted that the guidelines examined in this study were produced in relatively affluent countries with developed healthcare systems and so the use of laboratory medicine testing advocated in other, less wealthy, countries may well differ.

\section{References}

1. Hallworth MJ, Epner PL, Ebert C, Fantz CR, Higgins TN, Kilpatrick ES, et al. IFCC Task Force on the Impact of Laboratory Medicine on Clinical Management and Outcomes. Current evidence and future perspectives on the effective practice of patient-centered laboratory medicine. Clin Chem. 2015;61:589-9. https://doi.org/10.1373/ clinchem.2014.232629

2. Hallworth MJ. The "70\% claim": what is the evidence base? Ann Clin Biochem. 2011;48:487-8. https://doi.org/10.1258/ acb.2011.011177

3. Becich MJ. Information management: moving from test results to clinical information. Clin Leadersh Manag Rev. 2000;14:296-300.

4. Rohr UP, Binder C, Dieterle T, Giusti F, Messina CGM, Toerien $E$, et al. The value of in vitro diagnostic testing in medi-
Nonetheless, this does not preclude the same methodology as used here being applied to any alternative CVD guidelines from other countries.

Beyond CVD, there is also the possibility that the same objective approach to guidelines could be applied to determine the contribution of laboratory medicine in other, less prevalent, disease groups. Combining the findings from these different disease groups could potentially provide a more accurate overall assessment of the significance of laboratory medicine to healthcare in general.

In summary, this study has found a requirement for the use of laboratory testing in $73 \%$ to $94 \%$ of cardiovascular guidelines produced by five different organisations. It does not provide a direct link to improved patient outcomes but does provide an index to assess the potential value of laboratory medicine to healthcare which can complement other metrics.

\section{Acknowledgements}

I, Allan Hicks, would like to thank A/Prof Indu Singh, Dr Avinash Kundur and Dr Adrian Charles as my doctorate supervisors.

\section{Potential conflict of interest}

None declared. cal practice: a status report. PloS One. 2016;11:e0149856. https://doi.org/10.1371/journal.pone.0149856

5. World Health Organization. Cardiovascular diseases (CVDs) factsheet. Available at: https://www.who.int/en/news-room/fact-sheets/detail/cardiovascular-diseases-(cvds). Accessed April 26th 2020.

6. Bhatnagar $P$, Wickramasinghe $K$, Williams J, Rayner $M$, Townsend $N$. The epidemiology of cardiovascular disease in the UK 2014. Heart. 2015;101:1182-9. https://doi. org/10.1136/heartjnl-2015-307516

7. Wilkins E, Wilson L, Wickramasinghe K, Bhatnagar P, Leal J, Luengo-Fernandez $R$, et al. European Cardiovascular Disease Statistics 2017. Brussels: European Heart Network, 2017.

8. Benjamin EJ, Muntner P, Alonso A, Bittencourt MS, Callaway CW, Carson AP, et al. on behalf of the American Heart Asso- 
ciation Council on Epidemiology and Prevention Statistics Committee and Stroke Statistics Subcommittee. Heart disease and stroke statistics - 2019 update: a report from the American Heart Association. Circulation. 2019;139:e56e528.

9. Australian Institute of Health and Welfare. Cardiovascular Disease snapshot. Available at: https://www.aihw.gov.au/ reports/heart-stroke-vascular-disease/cardiovascular-health-compendium/contents/how-many-australians-havecardiovascular-disease. Accessed April 23rd 2020.

10. National Institute for Health and Care Excellence Guidance and advice list. Available at: https://www.nice.org.uk/guidance/published?type =apg,csg,cg,mpg,ph,sg,sc,dg, hst,ipg, mtg, as, ta. Accessed April 23rd 2020.

11. European Society of Cardiology Clinical guidelines. Available at: https://www.escardio.org/Guidelines/Clinical-Practice-Guidelines. Accessed April 23rd 2020.
12. American College of Cardiology guidelines. Available at: https://www.acc.org/guidelines\#doctype=Guidelines. Accessed April 23rd 2020.

13. Cardiac Society of Australia and New Zealand resources. Available at: https://www.csanz.edu.au/resources/. Accessed April 23rd 2020.

14. The Australian Heart Foundation clinical information. Available at: https://www.heartfoundation.org.au/for-professionals/clinical-information/. Accessed April 23rd 2020.

15. Khanji MY, Bicalho VVS, van Waardhuizen CN, Ferket $B S$, Petersen SE, Hunink MGM. Cardiovascular risk assessment. A systematic review of guidelines. Ann Intern Med. 2016;165:713-22. https://doi.org/10.7326/M16-1110 